\title{
Power system short-term load forecasting
}

Jingyao Wang School of Electric Power Engineering, North China Electric Power University, Baoding 071000,
China.

763491449@qq.com

Keywords: Short-term load forecasting, Multiple linear regression, Residual standard deviation

\begin{abstract}
In modern power system, the influence of meteorological factors on the load is increasingly prominent. In order to make the decision-making in power system more scientific, we should consider the meteorological factors, to improve the short-term load forecasting accuracy.

Due to the weather factors influencing the load are multiple, with the method of multiple linear regression analysis, we respectively deal with daily maximum load and daily minimum load and daily average load and the relationship between meteorological factors and regression analysis, to get the regression coefficients and residual standard deviation of equation. Combined with the regression coefficient, we get a different degree of the meteorological factors influence on the load, and determine the forecasting meteorological factors to improve the accuracy.
\end{abstract}

\section{Introduction}

Short-term load forecasting is the power load forecasting in the coming week, a day, one hour or even a shorter period of time, is the foundation of power system operation and analysis. Through regression analysis to determine meteorological factors to improve the prediction precision, it is of great decision significance in guarantying the power system.

\section{Notations}

\begin{tabular}{cc}
\hline Symbols & Notation \\
\hline$\beta_{0}, \beta_{1}, \cdots, \beta_{m}$ & Regression coefficient \\
$x_{0}, x_{1}, \cdots, x_{m}$ & Meteorological factors \\
$E_{n}$ & N order unit matrix \\
$Q$ & The sum of squared residuals \\
RMSE & Residual standard deviation \\
\hline
\end{tabular}

\section{Model}

In the modern power system, the influence of meteorological factors on the power system is increasingly prominent. For getting the relationship between the load and meteorological factors, we carry on the regression analysis. In order to take into consideration of the multiple factors, we used multivariate linear regression analysis of the algorithm $[1,2]$.

The establishment of the model.

The model for multiple linear regression analysis is

$$
\left\{\begin{array}{l}
y=\beta_{0}+\beta_{1} x_{1}+\cdots+\beta_{m} x_{m}+\varepsilon \\
\varepsilon \sim N\left(0, \sigma^{2}\right)
\end{array}\right.
$$

Now get $\mathrm{n}$ independent observation data $\left(\mathrm{y}_{\mathrm{i}}, \mathrm{x}_{\mathrm{i} 1}, \cdots, \mathrm{x}_{\mathrm{im}}\right), \mathrm{i}=1, \cdots, \mathrm{n}, \mathrm{n}>\mathrm{m}$

We can get 


$$
\left\{\begin{array}{l}
y_{i}=\beta_{0}+\beta_{1} x_{i 1}+\cdots+\beta_{m} x_{i m}+\varepsilon_{i} \\
\varepsilon_{i} \sim N\left(0, \sigma^{2}\right), i=1, \cdots, n
\end{array}\right.
$$

We design

$$
\begin{gathered}
X=\left[\begin{array}{cccc}
1 & x_{11} & \cdots & x_{1 m} \\
\vdots & \vdots & \cdots & \vdots \\
1 & x_{n 1} & \cdots & x_{n m}
\end{array}\right] \\
Y=\left[\begin{array}{c}
y_{1} \\
\vdots \\
y_{n}
\end{array}\right] \\
\varepsilon=\left[\begin{array}{lll}
\varepsilon_{1} & \cdots & \varepsilon_{n}
\end{array}\right]^{T} \\
\beta=\left[\begin{array}{llll}
\beta_{0} & \beta_{1} & \cdots & \beta_{m}
\end{array}\right]^{T}
\end{gathered}
$$

Arranging for

$$
\left\{\begin{array}{l}
Y=X \beta+\varepsilon \\
\varepsilon \sim N\left(0, \sigma^{2} E_{n}\right)
\end{array}\right.
$$

And matrix En is order n units.

\section{The solution of the model.}

Combined with the known data, we use least squares method for a estimate of $\beta_{0}, \beta_{1}, \beta_{2}, \beta_{3}$, $\beta_{4}, \beta_{5}$. So we should choose the estimate $\hat{\beta}_{j}$, Make the error sum of squares $\mathrm{Q}$ Is the smallest when $\beta_{j}=\hat{\beta}_{j}$.

Among them,

$$
Q=\sum_{i=1}^{25} \varepsilon_{i}^{2}=\sum_{i=1}^{25}\left(y_{i}-\hat{y_{i}}\right)^{2}=\sum_{i=1}^{n}\left(y_{i}-\beta_{0}-\beta_{1} x_{i 1}-\beta_{2} x_{i 2}-\beta_{3} x_{i 3}\right)^{2}
$$

Therefore, we design

$$
\frac{\partial Q}{\partial \beta_{j}}=0, j=0,1,2,3,4,5
$$

To solve the normal equations, we can get the estimate

$$
\left[\hat{\beta}_{0}, \hat{\beta}_{1}, \hat{\beta}_{2}, \hat{\beta}_{3}, \hat{\beta}_{4}, \hat{\beta}_{5}\right]=\left(X^{T} X\right)^{-1} X^{T} Y
$$

Using Matlab, we get the relationship between load and meteorological factors.

The regression equation of daily maximum load and meteorological factors regression equation are

$\hat{\beta}_{0}=5.6041, \hat{\beta}_{1=-0.0335,} \hat{\beta}_{2}=0.1301, \hat{\beta}_{3}=0.1058, \hat{\beta}_{4=-0.0129}, \hat{\beta}_{5}=0.0059$,

$\mathrm{RMSE}=31.4131$

The regression equation of daily minimum load and meteorological factors regression equation are

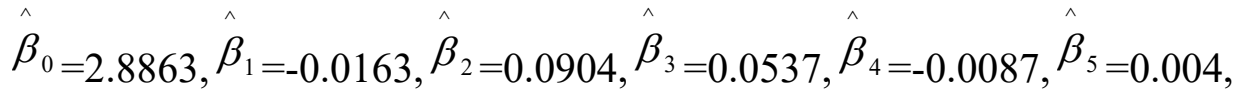

RMSE $=21.0531$

The regression equation of average daily load and meteorological factors regression equation are 


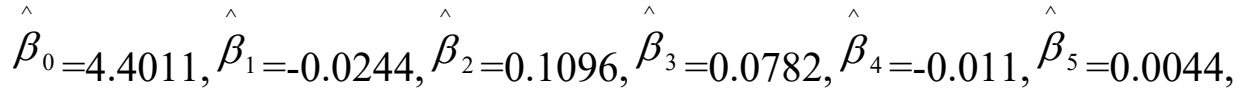

RMSE $=28.8059$

To observe the effects of meteorological factors on the load level, we made the influence of meteorological factors on the load diagram, as shown in Fig 1, Fig 2, Fig 3.
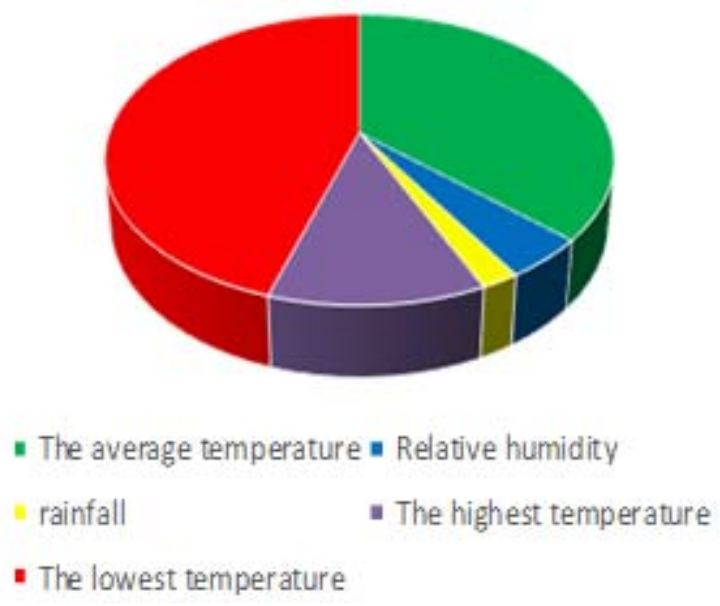

Fig 1 The relationship between daily maximum load and meteorological factors
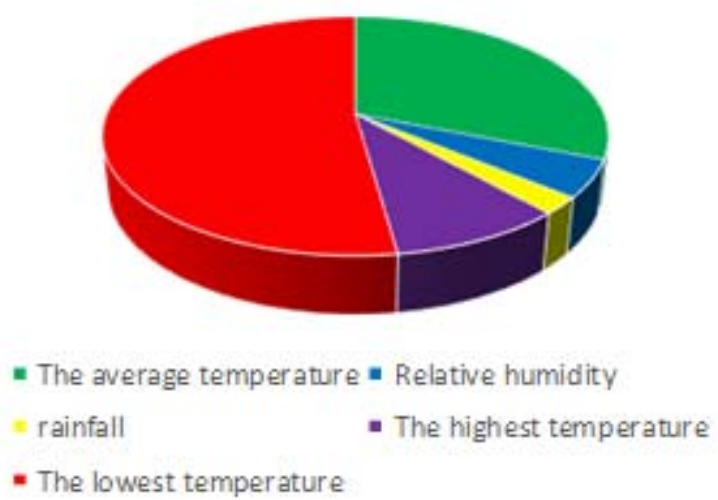

Fig 2 The relationship between daily minimum load and meteorological factors

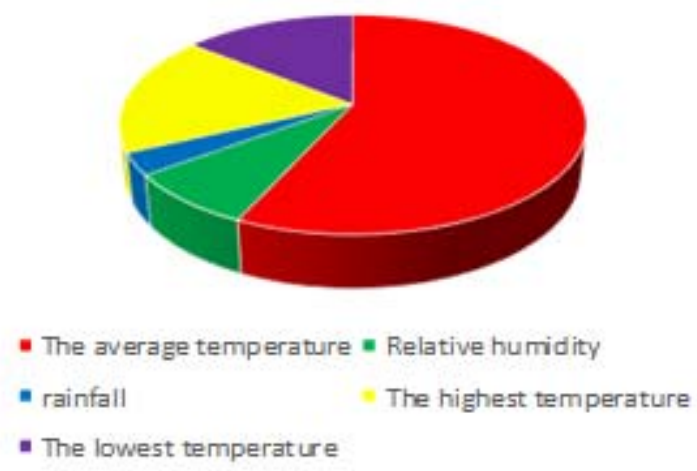

Fig 3 The relationship between average daily load and meteorological factors

Combined with image, we believe that for this region, we should give priority to the minimum temperature and average temperature, to improve the accuracy of load forecasting [3].

\section{Conclusions}

On the basis of the subject, we use the model to predict the result. Comparing to the climate factors, we found that the predicted results are more accurate, and the law is also used. This further illustrates the validity of the model. 


\section{References}

[1] Hailong Li. Considering the real-time meteorological factors of electric power system short-term load forecasting [D]. North China electric power university, 2015.

[2] Xiaoyan Li. Meteorological factors of electric power system short-term load forecasting research [D]. South China university of technology, 2013.

[3] Gang Mu, Kaiyuan Hou, Youhong Yang, Yongjie Hui, Kezhi Jiang. Study of load prediction of load regularity evaluation method [J]. Proceedings of the CSEE, 2001, 10:97-102. 